\title{
Designing Membrane-Active Nanoparticles: What are the Control Parameters?
}

\author{
Vladimir Baulin \\ Departament d'Enginyeria Quimica, Universitat Rovira i Virgili \\ 26 Av. dels Paisos Catalans, 43007 Tarragona, Spain \\ vladimir.baulin@urv.cat
}

Membrane-active molecules often undergo conformational changes while in contact with lipid membranes. Confined geometry of lipid bilayers imposes severe restrictions on the molecular structure of peptides and proteins interacting with bilayers. As these molecules move from solution to membrane-bound states there are dramatic changes in their structure and/or aggregation due to the two different chemical environments. In turn, change in shape and conformation of nanoobjects may induce severe changes in bilayer structure, ranging from modulation of membrane permeability to pore formation and even rupture of the bilayer. Thus, shape and size variation are often required for membrane activity of biomolecules, however the key property for membrane activity is amphiphilic nature of the compounds.

Using Single Chain Mean Field (SCMF) theory for lipid bilayers we show several examples of coupling between membrane activity and variation of conformation and polymers and nanoparticles demonstrate that hydrophobic objects embedded into the membrane can be used as lipid ion channels, which open and close pores in lipid bilayers by tuning their shape and size, permeation of lipid bilayers induced by polymers adsorbing at lipid bilayers. 